\title{
A utilização de joelheira na dor e função em pacientes com osteoartrite de joelho e síndrome da dor patelofemoral: uma revisão de escopo
}

The use of knee braces on pain and function in patients with knee osteoarthritis and patellofemoral pain syndrome: a scoping review

El uso de rodilleras sobre el dolor y la función en pacientes con osteoartritis de rodilla y síndrome de dolor femororrotuliano: una revisión del alcance

Adriano Carvalho de Oliveira ORCID: https://orcid.org/0000-0001-8225-7974 Universidade Federal do Amazonas, Brasil

E-mail: acofisioufam@gmail.com

Johrdy Amilton da Costa Braga

ORCID: https://orcid.org/0000-0003-2020-250X

Universidade Federal do Amazonas, Brasil

E-mail: johrdybraga@gmail.com

Neyvan Morais da Silva

ORCID: https://orcid.org/0000-0001-9352-4687

Universidade Federal do Amazonas, Brasil

E-mail: neyvanmorais@gmail.com

Vinicius Amaral Praia

ORCID: https://orcid.org/0000-0003-4561-1414

Universidade Federal do Amazonas, Brasil

E-mail: vkapraia@gmail.com

Carlos Júnior Silva de Souza

ORCID: https://orcid.org/0000-0002-3754-5536

Universidade Federal do Amazonas, Brasil

E-mail: karlosjss@gmail.com

Wendreson Barbosa Moraes

ORCID: https://orcid.org/0000-0002-5697-9137

Universidade Federal do Amazonas, Brasil

E-mail: wendreson.3@gmail.com

Thiago Santos da Silva

ORCID: https://orcid.org/0000-0001-8291-1470

Universidade Federal do Amazonas, Brasil

E-mail: thiagofisioufam@gmail.com

\begin{abstract}
Resumo
As desordens ortopédicas e/ou reumatológicas acometem com grande frequência o joelho, sendo a osteoartrite (OA) e a síndrome da dor patelofemoral (SDPF) as mais comuns entre essas desordens, e são os principais motivos pela busca por serviços médicos e fisioterapêuticos. Verificar os benefícios da utilização de joelheira na dor e na função do joelho em indivíduos com OA de joelho e a SDPF. Revisão de escopo elaborada conforme a metodologia da JBI. Foram considerados estudos envolvendo pacientes com OA de joelho e SDPF, sem restrição de sexo e raça, com idade $\geq 18$ anos, que utilizaram a joelheira como intervenção terapêutica, independentemente do tipo de joelheira, com os desfechos de dor e função ou funcionalidade, que foram publicados nos idiomas inglês, português e espanhol, com data de publicação de julho de 2001 a junho de 2021. As buscas por estudos publicados e literatura cinzenta foram conduzidos na PubMed, BVS, SciELO, PEDro, Catálogo de Teses e Dissertações da CAPES, Sumários.org e BDTD. A seleção dos estudos e apreciação crítica (pela escala PEDro) foram realizadas por dois revisores independentes. Foram incluídos seis estudos, com um total de 337 pacientes. Os estudos ajustaram o uso da joelheira entre 2 ou até 12 horas de utilização sem intervalo, durante 3 a 12 semanas. A utilização de joelheira apresenta benefícios em indivíduos portadores de osteoartrite de joelho e síndrome da dor patelofemoral, promovendo de alívio da dor e melhora da função do joelho. Aconselha-se o uso de joelheiras elásticas (e/ou articuladas), ajustáveis, com reforço patelar.
\end{abstract}

Palavras-chave: Dor; Modalidades de fisioterapia; Osteoartrite de joelho; Síndrome da dor patelofemoral.

\section{Abstract}

At disorders orthopedic and/or rheumatologic disorders frequently affect the knee, with osteoarthritis (OA) and patellofemoral pain syndrome (PFPS) being the most common among these disorders and are the main reasons for 
seeking medical and physical therapy services. To check the benefits of using a knee brace on pain and knee function in individuals with Knee OA and PFPS. Scope review prepared according to JBI methodology. Were considered studies involving patients with knee OA and SDPF, without restriction of sex and race, aged $\geq 18$ years old, who used the knee brace as therapeutic intervention, regardless of the type of knee brace, with the pain outcomes and function or functionality, which were published in English, Portuguese and Spanish, with publication date from July 2001 to June 2021. Searches for published studies and gray literature were conducted in PubMed, BVS, SciELO, PEDro, Catálogo de Teses e Dissertações da CAPES, Sumários.org and BDTD. Study selection and critical appraisal (using the PEDro scale) were performed by two independent reviewers. Were six studies were included, with a total of 337 patients. The studies adjusted the use of the knee brace between 2 or up to 12 hours of use without a break, for 3 to 12 weeks. Using a knee brace has benefits in individuals with knee osteoarthritis and patellofemoral pain syndrome, promoting pain relief and improvement in knee function. It is advisable to use elastic (and/or articulated), adjustable knee pads, with patellar reinforcement.

Keywords: Pain; Physical therapy modalities; Osteoarthritis knee; Patellofemoral pain syndrome.

\section{Resumen}

Los trastornos ortopédicos y / o reumatológicos afectan con frecuencia a la rodilla, siendo la osteoartritis (OA) y el síndrome de dolor patelofemoral (PFPS) los más comunes entre estos trastornos, y son los principales motivos para buscar servicios médicos y de fisioterapia. Verificar los beneficios de usar una rodillera sobre el dolor y la función de la rodilla en personas con Rodilla OA y PFPS. Revisión del alcance preparada según la metodología del JBI. Fueron considerados estudios en los que participaron pacientes con artrosis de rodilla y SDPF, sin restricción de sexo y raza, $\geq 18$ años, quien usó el rodillera como intervención terapéutica, independientemente del tipo de rodillera, con el resultados del dolor y función o funcionalidad, que se publicaron en inglés, portugués y español, con fecha de publicación de julio de 2001 a junio de 2021. Las búsquedas de estudios publicados y literatura gris se realizaron en PubMed, BVS, SciELO, PEDro, Catálogo de Teses e Dissertações da CAPES, Sumários.org y BDTD. La selección de estudios y la evaluación crítica (utilizando la escala PEDro) fueron realizadas por dos revisores independientes. Se incluyeron seis estudios, con un total de 337 pacientes. Los estudios ajustaron el uso de la rodillera entre 2 o hasta 12 horas de uso sin descanso, de 3 a 12 semanas. Usar una rodillera tiene beneficios en individuos con artrosis de rodilla y síndrome de dolor femororrotuliano, promoviendo alivio del dolor y mejora de la función de la rodilla. Es recomendable utilizar rodilleras elásticas (y / o articuladas), ajustables, con refuerzo rotuliano.

Palabras clave: Dolor; Modalidades de fisioterapia; Osteoartritis de la rodilla; Síndrome de dolor patelofemoral.

\section{Introdução}

As disfunções ortopédicas e/ou reumatológicas acometem frequentemente a articulação do joelho (Ghasemi; Dehghan, 2015; Yamamoto et al., 2019), sendo um fenômeno comum entre as pessoas, com frequência maior entre atletas e idosos (Chuang et al., 2007; Ghasemi; Dehghan, 2015; Melo et al., 2021). As formas mais comuns dessas disfunções são a osteoartrite (OA) (Chuang et al., 2007; Yamamoto et al., 2019; Vargas et al., 2020) e a síndrome da dor femoropatelar (SDPF) (Meira et al., 2012; Ghasemi; Dehghan, 2015).

A OA do joelho é uma condição inflamatória e degenerativa (Bryk et al., 2011; Schween; Gehring; Gollhofer, 2015; Yamada et al., 2018) que gera defeitos na integridade da cartilagem articular acompanhada de alterações no tecido ósseo (Oliveira et al., 2012). Sua prevalência tende a aumentar com o avançar da idade (Schween; Gehring; Gollhofer, 2015), afetando $25 \%$ dos indivíduos com idade superior a 18 anos (Chen et al., 2017) e mais de $80 \%$ das pessoas após os 65 anos (Charlier et al., 2016).

Já a SDPF, é uma desordem dolorosa que afeta principalmente a região anterior do joelho (Meira et al., 2012; Ghasemi; Dehghan, 2015), peri e retropatelar (Meira et al., 2012), que pode estar inicialmente associada a pequenas lesões na superfície articular. Com a progressão dessas lesões, a cartilagem se desgasta causando erosão do osso subcondral (Tavares et al., 2011; Ghasemi; Dehghan, 2015; Bersotti, 2018), esse desgaste precoce, além de gerar dor, pode ocasionar problemas como a condromalácia patelar (CP) e OA. Essa forma de comprometimento acomete $25 \%$ dos indivíduos maiores de 18 anos, sendo mais prevalente entre as pessoas fisicamente ativas, atletas, obesos, com predisposição pelo sexo feminino (Meira et al., 2012; Ghasemi; Dehghan, 2015; Chacur et al., 2017).

A AO do joelho e a SDPF, são dois dos principais motivos pela busca por serviços médicos e fisioterapêuticos (Ghasemi; Dehghan, 2015), seus sinais e sintomas clínicos são similares em sua maioria, apresentando dor, rigidez, fraqueza 
muscular, instabilidade e mudanças no alinhamento articular, provocando diminuição de habilidades funcionais essenciais, como sentar e levantar, subir e descer escadas, ajoelhar-se, andar e até mesmo em ficar de pé, podendo ainda aumentar a probabilidade de quedas (Bryk et al., 2011; Oliveira et al., 2014; Ghasemi; Dehghan, 2015; Yamamoto et al., 2019).

Inúmeras são as formas de tratamento para AO do joelho e a SDPF, podendo essas condições serem tratadas por meio de fármacos e cirurgias, no entanto, dependendo do estágio da lesão, o mais recomendado é o tratamento conservador, com destaque para a fisioterapia. Dentre os recursos fisioterapêuticos destacam-se os exercícios aeróbicos e fortalecimento muscular (Bryk et al., 2011; Ghasemi; Dehghan, 2015). Contudo, as limitações causadas por estas condições inviabilizam a prescrição desses exercícios de forma independente, pois na maioria das vezes são dolorosos e ineficazes (Callaghan; Parkes; Felson, 2016; Yamamoto et al., 2019).

$\mathrm{Na}$ tentativa de diminuir os impactos dessas condições, diversos recursos vêm sendo utilizados, destacando-se a joelheira (Bryk et al., 2011), uma vez que, vem se mostrando eficaz no controle da dor e melhora da função durante a realização dos exercícios (Chuang et al., 2007; Bryk et al., 2011; Ghasemi; Dehghan, 2015; Schween; Gehring; Gollhofer, 2015; Callaghan; Parkes; Felson, 2016; Yamamoto et al., 2019). Porém, existem divergências enquanto a sua contribuição e eficácia na melhora da função, alinhamento patelar e alívio da dor (Callaghan; Parkes; Felson, 2016; Yamamoto et al., 2019). Dessa forma, surge o seguinte questionamento: Quais os benefícios da utilização de joelheira na dor e na função do joelho em pacientes com OA de joelho e SDPF?

Sendo assim, esta revisão tem como objetivo verificar os benefícios da utilização de joelheira na dor e na função do joelho em indivíduos com OA de joelho e SDPF.

\section{Metodologia}

Trata-se de uma revisão de escopo, elaborada em conformidade com a metodologia do JBI Collaboration (JBI) (Peters et al., 2020), e relatada de acordo com o PRISMA Extension for Scoping Reviews: Checklist and Explanation (PRISMA-ScR) (Tricco et al., 2020).

\subsection{Perguntas de revisão}

Quais os benefícios da utilização de joelheira na dor e na função do joelho em pacientes com OA de joelho e SDPF?

\subsection{Critérios de elegibilidade}

Seguindo a recomendação do JBI, os critérios de elegibilidade foram elaborados baseados no mnemônico PCC (participantes, conceito e contexto) (Peters et al., 2020).

\subsubsection{Participantes}

Considerou-se, nesta revisão, estudos envolvendo pacientes com OA de joelho e SDPF, sem restrição de sexo e raça, com idade $\geq 18$ anos.

\subsubsection{Conceito}

Esta revisão considerou estudos relacionados à utilização de joelheira como intervenção terapêutica de indivíduos com OA de joelho e SDPF, independentemente do tipo de joelheira que fora utilizada (de faixa simples, elástica, órtese; convencional ou articulada).

Foram considerados os desfechos de dor e função ou funcionalidade, avaliados com instrumentos de medida padronizados e com boas estimativas de validade. 


\subsubsection{Contexto}

Foram considerados estudos conduzidos em quaisquer contextos clínicos e em quaisquer contextos geográficos.

\subsection{Tipos de estudos}

Foram considerados nesta revisão ensaios clínicos randomizados, ensaios clínicos controlados, ensaios clínicos pragmáticos e estudos quase-experimentais ou o tipo antes e depois, publicados nos idiomas inglês, português e espanhol (sendo estes idiomas compreendidos pelos revisores), com data de publicação de julho de 2001 a junho de 2021 . O período de busca foi ajustado para 20 (vinte) anos, visando localizar apenas estudos mais recentes.

\subsection{Estratégia de busca}

Buscas preliminares foram realizadas nas bases de dados PubMed e PEDro com objetivo de verificar estudos potencialmente elegíveis e explorar as palavras-chaves e termos de índice que eles utilizavam para construir uma estratégia de busca (sintaxe da revisão) [elastic knee sleeve AND (osteoarthritis OR patellofemoral pain syndrome OR chondromalacia patellae) AND (knee Joint OR knee)]. As buscas foram realizadas nos bancos de dados na primeira quinzena do mês de junho de 2021. A estratégia de busca desenvolvida foi adaptada de acordo com cada banco de dados incluído na revisão, registradas no Quadro 1.

Quadro 1. Resultado da estratégia de busca nos bancos de dados.

\begin{tabular}{|c|c|c|}
\hline Base de dados & $\begin{array}{c}\text { Itens } \\
\text { encontrados }\end{array}$ & Busca (Consulta) \\
\hline PubMed & 183.915 & 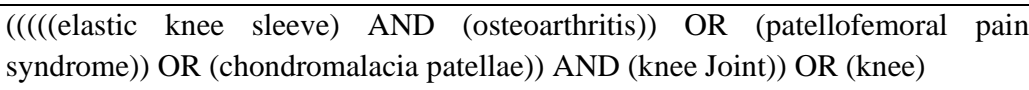 \\
\hline \multirow{3}{*}{$\begin{array}{l}\text { Biblioteca Virtual } \\
\text { de Saúde (BVS) }\end{array}$} & 0 & $\begin{array}{l}\text { (joelheira) AND (osteoartrite) OR (osteoartrose) OR (gonartrose) OR (artrose) } \\
\text { OR (síndrome da dor femoropatelar) OR (condromalacia da patela) AND } \\
\text { (articulação do joelho) OR (joelho) }\end{array}$ \\
\hline & 17 & $\begin{array}{l}\text { (elastic knee sleeve) AND (osteoarthritis) OR (patellofemoral pain syndrome) } \\
\text { OR (chondromalacia patellae) OR (knee Joint) OR (knee) }\end{array}$ \\
\hline & 0 & $\begin{array}{l}\text { (rodillera elástica) AND (osteoartritis) OR (síndrome de dolor patelofemoral) OR } \\
\text { (condromalacia de la rótula) OR (articulación de la rodilla) OR (rodilla) }\end{array}$ \\
\hline \multirow{3}{*}{$\begin{array}{l}\text { Scientific Electronic } \\
\text { Library Online } \\
\quad \text { (SciELO) }\end{array}$} & 0 & $\begin{array}{l}\text { (joelheira) AND (osteoartrite) OR (osteoartrose) OR (gonartrose) OR (artrose) } \\
\text { OR (síndrome da dor femoropatelar) OR (condromalacia da patela) AND } \\
\text { (articulação do joelho) OR (joelho) }\end{array}$ \\
\hline & 1 & $\begin{array}{l}\text { (elastic knee sleeve) AND (osteoarthritis) OR (patellofemoral pain syndrome) } \\
\text { OR (chondromalacia patellae) OR (knee Joint) OR (knee) }\end{array}$ \\
\hline & 0 & $\begin{array}{l}\text { (rodillera elástica) AND (osteoartritis) OR (síndrome de dolor patelofemoral) OR } \\
\text { (condromalacia de la rótula) OR (articulación de la rodilla) OR (rodilla) }\end{array}$ \\
\hline \multirow{3}{*}{$\begin{array}{l}\text { Physiotherapy } \\
\text { Evidence Database } \\
\quad \text { (PEDro) }\end{array}$} & 2 & elastic* knee* $^{*}$ sleeve* osteoarthritis* knee* \\
\hline & 0 & elastic* knee* sleeve* patellofemoral* pain* syndrome* knee* \\
\hline & 0 & elastic* knee* $^{*}$ sleeve* chondromalacia* patellae* knee* $^{*}$ \\
\hline $\begin{array}{l}\text { Catálogo de Teses e } \\
\text { Dissertações da }\end{array}$ & 0 & $\begin{array}{l}\text { joelheira AND osteoartrite OR osteoartrose OR gonartrose OR artrose OR } \\
\text { síndrome da dor femoropatelar OR condromalacia da patela AND articulação do }\end{array}$ \\
\hline
\end{tabular}


Research, Society and Development, v. 10, n. 14, e190101421700, 2021

(CC BY 4.0) | ISSN 2525-3409 | DOI: http://dx.doi.org/10.33448/rsd-v10i14.21700

\begin{tabular}{|c|c|c|}
\hline \multirow[t]{2}{*}{ CAPES - Brasil } & & joelho OR joelho \\
\hline & 0 & $\begin{array}{l}\text { elastic knee sleeve AND osteoarthritis OR patellofemoral pain syndrome OR } \\
\text { chondromalacia patellae OR knee Joint OR knee }\end{array}$ \\
\hline \multirow{2}{*}{$\begin{array}{l}\text { Sumários de } \\
\text { Revistas Brasileiras } \\
\text { (Sumários.org) }\end{array}$} & 0 & $\begin{array}{l}\text { joelheira AND osteoartrite OR osteoartrose OR gonartrose OR artrose OR } \\
\text { síndrome da dor femoropatelar OR condromalacia da patela AND articulação do } \\
\text { joelho OR joelho }\end{array}$ \\
\hline & 0 & $\begin{array}{l}\text { elastic knee sleeve AND osteoarthritis OR patellofemoral pain syndrome OR } \\
\text { chondromalacia patellae OR knee Joint OR knee }\end{array}$ \\
\hline \multirow{2}{*}{$\begin{array}{l}\text { Biblioteca Digital } \\
\text { Brasileira de Testes } \\
\text { e Dissertações } \\
\text { (BDTD) }\end{array}$} & 0 & $\begin{array}{l}\text { "(Todos os campos:joelheira E Todos os campos:osteoartrite E Todos os } \\
\text { campos:osteoartrose E Todos os campos:gonartrose E Todos os campos:artrose E } \\
\text { Todos os campos:síndrome da dor femoropatelar E Todos os } \\
\text { campos:condromalacia da patela E Todos os campos:articulação do joelho E } \\
\text { Todos os campos:joelho)" }\end{array}$ \\
\hline & 0 & $\begin{array}{l}\text { "(Todos os campos:elastic knee sleeve E Todos os campos:osteoarthritis E Todos } \\
\text { os campos:patellofemoral pain syndrome E Todos os campos:chondromalacia } \\
\text { patellae E Todos os campos:knee Joint E Todos os campos:knee)" }\end{array}$ \\
\hline
\end{tabular}

Fonte: Autores (2021).

\subsection{Fontes de informação}

A busca teve como objetivo localizar estudos publicados e de literatura cinzenta. As fontes de estudos publicados foram a Pubmed, BVS, SciELO e PEDro. Fontes de literatura cinzenta foram a Catálogo de Teses e Dissertações da CAPES CAPES (CTD), Sumários.org e BDTD. As buscas foram realizadas de forma autônoma por dois pesquisadores. Casos em que o texto completo do estudo estava indisponível, os autores foram contatados e quando não foi obtido resposta os estudos foram excluídos.

\subsection{Seleção dos estudos}

Inicialmente, uma seleção preliminar baseada na verificação do título e resumo dos estudos potencialmente elegíveis foi realizada. Posteriormente, o texto completo dos estudos pré-selecionados foi avaliado seguindo rigorosamente os critérios de inclusão e exclusão estabelecidos nesta pesquisa.

\subsection{Análise da qualidade metodológica}

Apesar de não ser próprio de revisões de escopo realizar avaliação da qualidade metodológica dos estudos, esta etapa foi realizada na presente revisão visando selecionar apenas estudos com boa qualidade metodológica para a síntese dos dados. Para isto, foi utilizada a Escala de PEDro, versão traduzida para o português, a qual foi desenvolvia pela Physiotherapy Evidence Database e que possui 11 itens associados a critérios de avaliação e análise da metodologia, os quais são aplicados em estudos experimentais tendo uma pontuação que totaliza 10 pontos (Shiwa et al., 2011).

A apreciação crítica dos estudos de acordo com a Escala de PEDro foi realizada por dois revisores independentes. As divergências entre os revisores foram resolvidas mediante a discussão, e quando a consonância não foi alcançada foi considerada a opinião de um terceiro revisor.

Após a análise, os estudos foram classificados de acordo sua pontuação na Escala PEDro: estudos com escores entre 0 a 3 foram classificados com baixa qualidade metodológica; de 4 a 7 , qualidade metodológica moderada; e de 8 a 10, boa 
qualidade metodológica. Todavia, somente estudos com escore PEDro igual ou superior a 3 foram considerados para a extração e síntese dos dados.

\subsection{Extração de dados}

A extração de dados dos artigos selecionados foi realizada com uma ferramenta desenvolvida pelos revisores (Tabela 1). As informações extraídas foram: autores e ano de publicação, objetivo, participantes (condição patológica, número de participantes, faixa etária, sexo) desenho do estudo, intervenção (caracterização do tratamento), resultados e conclusões. Foi utilizado o software Microsoft Word (C) 2013 para a tabulação dos dados.

\subsection{Apresentação dos achados}

Os resultados obtidos foram descritos por meio de síntese descritiva e tabelas, em conformidade com a pergunta e objetivo da revisão.

\section{Resultados}

Foram inclusos nesta revisão seis estudos, com um total de 337 pacientes. Na Figura 1, existe um fluxograma demonstrando o número de bancos de dados rastreados, os estudos avaliados para a elegibilidade e incluídos na revisão, com razões de exclusão dos estudos.

Figura 1. Fluxograma demonstrando o processo de busca e seleção dos estudos.

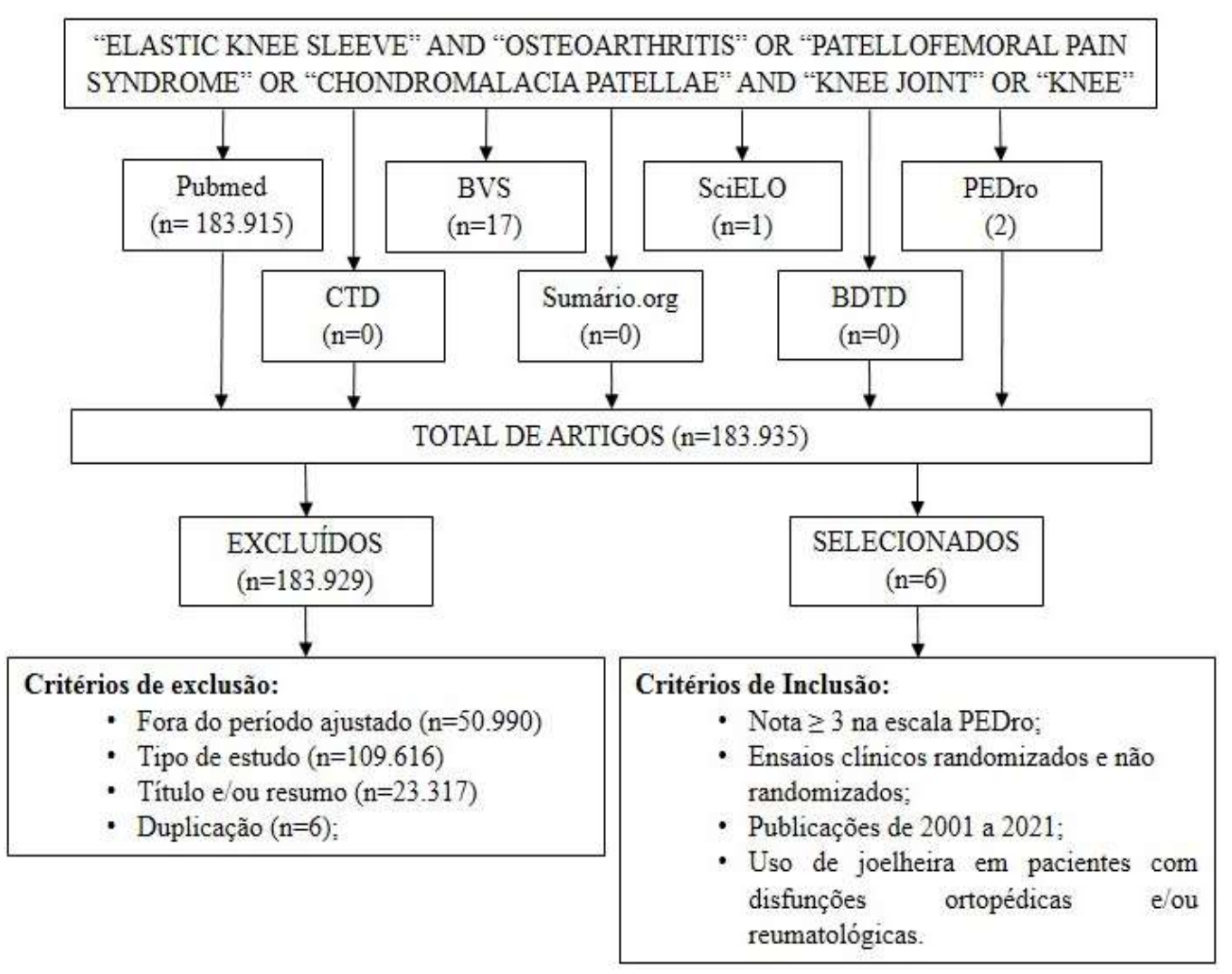

Fonte: Autores (2021).

Os estudos e seus respectivos protocolos terapêuticos estão descritos na Tabela 1. 
Tabela 1 - Resumo das evidências da utilização de joelheira na dor e na função em pacientes com osteoartrite e síndrome da dor patelofemoral.

\begin{tabular}{|c|c|c|c|c|c|c|}
\hline $\begin{array}{l}\text { Autor(es) } \\
\text { /Ano }\end{array}$ & Objetivo & Desenho/Amostra & $\begin{array}{l}\text { Metodologia } \\
\text { Intervenção }\end{array}$ & Resultados & Conclusão & $\begin{array}{l}\text { Escore } \\
\text { PEDro }\end{array}$ \\
\hline $\begin{array}{l}\text { Bryk et al. } \\
\text { (2011) }\end{array}$ & $\begin{array}{l}\text { Analisar a eficácia } \\
\text { imediata da joelheira } \\
\text { elástica na dor e na } \\
\text { capacidade funcional } \\
\text { em indivíduos com } \\
\text { OA de joelho. }\end{array}$ & $\begin{array}{l}\text { A amostra com } 74 \\
\text { indivíduos, com } \\
\text { idade média de } 58 \\
\text { anos, e dor acima } \\
\text { de três pontos na } \\
\text { escalar visual } \\
\text { analógica (EVA) e } \\
\text { no mínimo quatro } \\
\text { itens dos critérios } \\
\text { clínicos segundo o } \\
\text { American College } \\
\text { of Rheumatology. }\end{array}$ & $\begin{array}{l}\text { Os pacientes foram alocados em } \\
\text { dois grupos com e sem joelheira. } \\
\text { Foram orientados a realizar os } \\
\text { testes uma única vez antes do início } \\
\text { das avaliações, para aprendizado; A } \\
\text { capacidade funcional e a dor foram } \\
\text { analisados por meio dos testes Stair } \\
\text { Climb Power Test (SCPT), Timed } \\
\text { Up and Go (TUG), Caminhada de } 8 \\
\text { Metros (C8M) e a EVA. }\end{array}$ & $\begin{array}{l}\text { Foi encontrada redução da dor nos } \\
\text { pacientes do grupo que utilizou a } \\
\text { joelheira (EVA - P < 0,001), assim } \\
\text { como melhoras na capacidade } \\
\text { funcional que foi evidenciada pela } \\
\text { melhora na dor, inflamação, } \\
\text { limitação da amplitude de } \\
\text { movimento e rigidez articular } \\
\text { (Testes C8M e TUG - P }<0,05 \text { ). }\end{array}$ & $\begin{array}{l}\text { A joelheira elástica foi } \\
\text { eficiente na melhora } \\
\text { imediata da capacidade } \\
\text { funcional e da dor, e pode } \\
\text { auxiliar e/ou facilitar a } \\
\text { execução de exercícios } \\
\text { terapêuticos. }\end{array}$ & $6 / 10$ \\
\hline $\begin{array}{l}\text { Ghasemi \& } \\
\text { Dehghan. } \\
\text { (2015) }\end{array}$ & $\begin{array}{lr}\text { Determinar } & \text { a } \\
\text { eficácia da órtese } \\
\text { palumbo } & \text { de } \\
\text { neoprene e da órtese } \\
\text { estável Genu direxa } \\
\text { na dor e r nas } \\
\text { atividades de vida } \\
\text { diária (AVD). }\end{array}$ & $\begin{array}{l}\text { Amostra com } 30 \\
\text { homens, com } \\
\text { idade entre } 18 \text { e } 40 \\
\text { anos, e diagnóstico } \\
\text { de SDPF. }\end{array}$ & $\begin{array}{l}\text { Os participantes foram alocados em } \\
\text { dois grupos, os que usaram a órtese } \\
\text { palumbo de neoprene e os que } \\
\text { usaram a órtese estável Genu } \\
\text { direxa, durante } 3 \text { semanas. Foram } \\
\text { instruídos sobre como colocar e } \\
\text { tirar a órtese, sendo orientados a } \\
\text { tirá-la apenas na hora de dormir. } \\
\text { Eles não receberam nenhuma outra } \\
\text { forma de tratamento além da } \\
\text { joelheira; A dor e a AVD foram } \\
\text { avaliadas por meio da EVA e de um } \\
\text { questionário modificado para }\end{array}$ & $\begin{array}{l}\text { Ambos os grupos apresentaram } \\
\text { melhora significativa na redução da } \\
\text { dor e aumento nas AVD após o uso } \\
\text { da joelheira. Na comparação das } \\
\text { variáveis entre os grupos, não } \\
\text { foram encontradas diferenças } \\
\text { significativas entre a intensidade da } \\
\text { dor e as AVD ( } \mathrm{p}=0,592, \mathrm{p}= \\
0,887 \text { ). Em ambos os grupos, a } \\
\text { média da intensidade da dor foi } \\
\text { diferente antes, durante e após o } \\
\text { uso da joelheira (p <0,05), sendo } \\
\text { que a mesma ainda permitiu }\end{array}$ & $\begin{array}{l}\text { Os resultados deste estudo } \\
\text { indicaram que as órteses } \\
\text { estáveis palumbo e Genu } \\
\text { direxa melhoraram os } \\
\text { sinais e sintomas da SDPF, } \\
\text { principalmente os } \\
\text { referentes a intensidade da } \\
\text { dor e AVD. }\end{array}$ & $6 / 10$ \\
\hline
\end{tabular}


pacientes com OA e lesões no aumentar o nível de atividades, joelho. A intensidade da dor, AVD amplitude de movimento e redução e rigidez articular foram analisadas da rigidez articular.

antes do tratamento e após 3

semanas de tratamento.

Callaghan, Investigar os efeitos Ensaio Clínico Os participantes foram alocados em

Parkes \& de uma joelheira Randomizado, dois grupos com e sem joelheira. dife

Felson.

(2016)

flexível na contração

voluntária máxima

(CVM) na inibição

muscular artrogênica

(IMA) do quadríceps

em pacientes com

AO da articulação patelofemoral.

seguido por um Não receberam nenhuma outra

Ensaio Open-

label;

Open- forma de tratamento além da joelheira e seus analgésicos regulares. O uso da joelheira BioSkin com orifício para patela, A amostra foi composta por 108 individuos, com idade entre 40 e 70 anos, e pelo menos 3 meses de dor com pontuação de Kellgren-

Lawrence de 2 ou

3 para OA. seguida, todos foram sbmetidos ao $<0,01)$. Embora estatisticamente uso da jolheira por mais 12 significativos, esses resultados têm semanas; A CVM e IM do significado clínico duvidoso. quadríceps foram medidas após 6 e 12 semanas.

Após as 6 semanas, a CVM não A joelheira não gerou (h) diárias, durante 6 semanas. Em 0,004) e diminuição da IAM (P músculo quadríceps.

Os pacientes foram alocados no grupo controle (joelheira neoprene com orifício para patela) e grupo de estudo (joelheira funcional neoprene com tubos de borracha na articulação patelofemoral, em comparação com uma joelheira neoprene com orifício para patela, em pacientes com OA da articulação

Estudo Prospectivo,

Randomizado;

$A$ A amostra foi composta por 57 indivíduos, com

idade maior ou igual a 30 anos, e diagnostico de $\mathrm{AO}$ parte superior, inferior e lateral da patela). Eles foram orientados sobre a OA e suas formas de tratamento, e a usar a joelheira por $2 \mathrm{~h}$ no primeiro dia e aumentar em meia hora por dia a partir do segundo dia, diferiu entre os grupos $(\mathrm{P}=0,20)$, nquanto que a IMA diminuiu significativamente no grupo com oelheira $(\mathrm{P}=0,002)$. Já após as 12 semanas, todos os participantes que usaram a joelheira flexível, reduzindo a inibição do

efeitos adversos no músculo quadríceps femoral em pacientes com OA da articulação patelofemoral, aumentando modestamente a força e

Ambos os grupos obtiveram Ambas as joelheiras melhoras nas condições de dor, melhoraram a dor, a rigidez e função sem diferença função e alteraram o significativa entre os grupos. O consumo de medicamentos consumo de medicamentos somente no primeiro mês. diminuiu em ambos os grupos no A joelheira funcional primeiro mês, aumentando no neoprene com tubos de terceiro mês. O consumo de borracha na parte superior, naproxeno foi progressivamente inferior e lateral da patela maior no grupo controle $(\mathrm{P}=$ propiciou analgesia sem $\mathrm{O}$ aumento do consumo de 
patelofemoral.

(critérios clínicos até o máximo de 12h/dia, podendo da ACR8 para dor as $12 \mathrm{~h}$ serem contínuas ou em e sensibilidade).

intervalos (inicialmente $\geq 4 \mathrm{~h} \mathrm{e} \mathrm{a}$ parte da segunda semana $\leq 2 \mathrm{~h}$ )

Foram avaliados as circunferências da coxa e da perna, assim como a dor, rigidez, função e uso de drogas por meio do questionário Lequesne, testes de senta e levanta, TUG e caminhada de seis minutos, antes da colocação da joelheira e após 1 e 3 meses.

Chuang et al. (2007)

Investigar os efeitos Ensaio das joelheiras no Controlado; equilíbrio estático e dinâmico em pacientes com OA de joelho.
Os

icipantes foram alocados e dois grupos. No grupo $\mathrm{A}$ inicialmente, os indivíduos foram orientados a realizar os testes de A amostra foi equilíbrio sem a joelheira, porém,
composta por 50 posteriormente, os mesmos testes indivíduos po fo for ambos os sexos, com idade entre 40 e 78 anos, e diagnostico de OA de acordo com os critérios

da

American College of Rheumatology. fizeram apenas o procedimento inverso. Os testes de equilíbrio foram realizados de forma estática e dinâmica, sendo que os todos os sujeitos tiveram três tentativas consecutivas para completar os testes; Os escores de equilíbrio

\section{$0,011)$.}

naproxeno.
Os resultados revelaram uma Usar joelheiras é eficaz pontuação significativamente durante a realização de menor nos pacientes quando atividades estáticas e utilizaram a joelheira neoprene ( $\mathrm{p}$ dinâmicas em portadores $<0,05)$. Pontuações mais baixas de OA de joelho. Assim, a indicaram melhor desempenho de joelheira neoprene se torna equilíbrio, ou seja, quanto mais uma alternativa no próxima do zero a pontuação era, tratamento da OA de melhor era o equilíbrio. $\mathrm{O}$ achado joelho. neoprene. Os indivíduos do grupo B foram avaliados utilizado um o software KAT 2000, que quantificou o desempenho do controle motor dos membros inferiores. deste estudo demonstrou que

pacientes com OA de joelho usando joelheira podem experimentar aumento da capacidade de equilíbrio em condições estáticas e dinâmicas. A melhora aumenta a sensação de segurança durante as atividades físicas e consequentemente pode evitar que pacientes caiam, diminuindo o risco de queda. 


\begin{tabular}{|c|c|c|c|c|c|}
\hline $\begin{array}{l}\text { Gehring \& } \\
\text { Gollhofer. } \\
\text { (2015) }\end{array}$ & $\begin{array}{l}\text { imediatos de uma } \\
\text { joelheira elástica na } \\
\text { cinemática e cinética } \\
\text { da marcha no plano } \\
\text { frontal } \\
\text { indivíduos com } \\
\text { de joelhos. }\end{array}$ & $\begin{array}{l}\text { A amostra foi } \\
\text { composta por } 18 \\
\text { indivíduos } \\
\text { ambos os sexos, } \\
\text { com idade entre } 40 \\
\text { e } 78 \text { anos, e } \\
\text { diagnostico de OA } \\
\text { na porção medial } \\
\text { da articulação do } \\
\text { joelho. }\end{array}$ & $\begin{array}{l}\text { dois grupos com e sem joelheira. } \\
\text { Os mesmos foram orientados a } \\
\text { realizar caminhadas retas de cerca } \\
\text { de } 10 \text { metros, utilizando sapatos } \\
\text { padronizados com baixo } \\
\text { amortecimento e sem palmilhas } \\
\text { personalizadas durante todo o } \\
\text { experimento para evitar que os } \\
\text { efeitos potenciais relacionados ao } \\
\text { calçado influenciem a análise; A } \\
\text { análise cinemática e cinética foi } \\
\text { realizada a uma frequência de } \\
\text { amostragem de } 200 \mathrm{~Hz} \text { usando um } \\
\text { sistema de análise de movimento } \\
\text { 3D (Software Vicon). }\end{array}$ & $\begin{array}{l}\text { caminhadas, o ângulo de adução do } \\
\text { joelho no contato com o solo foi } \\
\text { reduzido em } 1,9 \pm 2,1^{\circ}(\mathrm{P}=0,006) \text {. } \\
\text { O pico de adução do joelho foi } \\
\text { reduzido em } 1,5 \pm 1,6^{\circ}(\mathrm{P}=0,004) \text {. } \\
\text { O primeiro pico de momento de } \\
\text { adução do joelho e o impulso } \\
\text { positivo de adução do joelho foram } \\
\text { diminuídos em } 10,1 \% \text {. Esses } \\
\text { desfechos favorecem a utilização da } \\
\text { joelheira, uma vez que, um maior } \\
\text { grau de adução do joelho no } \\
\text { momento da caminhada é um fator } \\
\text { de risco para a progressão da OA } \\
\text { medial do joelho. }\end{array}$ & $\begin{array}{l}\text { elástica durante a } \\
\text { caminhada pode reduzir o } \\
\text { ângulo, momentos } \\
\text { impulso de adução do } \\
\text { joelho em indivíduos com } \\
\text { OA de joelho. }\end{array}$ \\
\hline
\end{tabular}

Legenda: EVA: Escala visual analógica; SCPT: Stair Climb Power Test; TUG: Timed Up and Go, C8M: Caminhada de 8 Metros; AVD: Atividades de vida diária; CVM: Contração voluntária Fonte: Autores (2021) 


\subsection{Síntese dos achados descritos na Tabela 1}

Identificou-se dentre os estudos, além da utilização da joelheira, o uso de medicamentos, os quais foram empregados no controle das disfunções geradas pela OA de joelho. Os estudos ajustaram o uso da joelheira para que fosse possível a avaliação dos seus efeitos imediatos e após 2 ou até 12 horas de utilização sem intervalo, durante 3 a 12 semanas (valores máximos e mínimos de acordo com os estudos).

Os protocolos de utilização da joelheira adotados pelos estudos apresentaram efeito positivo nos impactos ocasionados pela OA de joelho e da SDPF, melhorando a função do joelho, capacidade de realização de atividades de vida diária, capacidade funcional, capacidade contração voluntária máxima, o equilíbrio em condições estáticas e dinâmicas, aumentando a amplitude de movimento, diminuindo a inibição muscular artrogênica, rigidez articular, dor, risco de queda, ajudando no controle da inflamação e além disso, ainda reduz o ângulo e picos de adução do joelho que são um fator de risco para a progressão dessas.

\section{Discussão}

Todos os estudos evidenciaram a utilização da joelheira nas disfunções causadas pela da OA de joelho e da SDPF (Chuang et al., 2007; Bryk et al., 2011; Ghasemi; Dehghan, 2015; Schween; Gehring; Gollhofer, 2015; Callaghan; Parkes; Felson, 2016; Yamamoto et al., 2019). Porém, os estudos avaliaram os efeitos da joelheira em condições diferentes, Chuang et al (2007), Bryk et al (2011) e Schween, Gehring e Gollhofer (2015), avaliaram o seu efeito imediata, enquanto que, Ghasemi e Dehghan (2015), Callaghan, Parkes e Felson (2016) e Yamamoto et al (2019), avaliaram o seu efeito após no mínimo 2 horas podendo chegar até 12 horas de utilização sem intervalo, durante entre 3 a 12 semanas.

Chuang et al (2007), investigaram os efeitos imediatos da joelheira no equilíbrio, e observaram o aumento da capacidade de equilíbrio em condições estáticas e dinâmicas. E não só isso, demostraram que o aumento da sensação de segurança durante as atividades físicas estáticas e dinâmicas podem evitar que pacientes caiam, e consequentemente diminuem o risco de queda. Corroborando com Schween, Gehring e Gollhofer (2015), que avaliaram os efeitos imediatos de uma joelheira elástica na cinemática e cinética da marcha, e evidenciaram que a joelheira melhora a estabilidade e reduz o ângulo e picos de adução do joelho, e diminuem o risco de progressão dessas patologias. Além disso, Bryk et al (2011), ao analisarem o efeito imediata da joelheira elástica na dor e na capacidade funcional, confirmaram que a joelheira é eficaz na melhora da capacidade funcional, já que, melhora os escores de dor, inflamação, limitação da amplitude de movimento e rigidez articular.

Callaghan, Parkes e Felson (2016), investigaram os efeitos de uma joelheira flexível na contração voluntária máxima e na inibição muscular artrogênica do quadríceps durante 12 semanas, e observaram que, os indivíduos que utilizaram a joelheira obtiveram aumento da contração voluntária máxima e diminuição da inibição muscular artrogênica do quadríceps. Alem do mais, Ghasemi e Dehghan (2015), compararam o efeito da órtese palumbo de neoprene e da órtese estável Genu direxa na dor e nas atividades de vida diária durante 3 semanas, sendo que as joelheiras eram retiradas apenas na hora de dormir, e não identificaram diferenças significativas entre as mesmas, mas evidenciaram melhoras no nível de atividades de vida diária, amplitude de movimento e redução da rigidez articular. Corroborando com Yamamoto et al (2019), que verificaram o efeito de uma órtese destinada a estabilizar a articulação patelofemoral, em comparação com uma joelheira neoprene com orifício para patela durante 12 semanas, sendo que os pacientes foram orientados a usar a joelheira por $2 \mathrm{~h}$ no primeiro dia e aumentar em meia hora por dia a partir do segundo dia, até o máximo de 12h/dia, e observaram melhoras nas condições de dor, rigidez e função, mas não identificaram diferença significativa entre as joelheiras.

A maioria dos estudos utilizaram a joelheira de forma isolada no tratamento da OA de joelho e da SDPF (Chuang et al., 2007; Bryk et al., 2011; Ghasemi; Dehghan, 2015; Schween; Gehring; Gollhofer, 2015), mas Callaghan; Parkes; Felson, 2016 e Yamamoto et al., 2019, utilizam a joelheira associado ao uso de analgésicos regulares, objetivando o controle das 
disfunções geradas pela OA de joelho, sendo que Yamamoto et al., 2019, evidenciaram em seu trabalho a diminuição do consumo desses medicamentos.

Baseado na declaração PRISMA-ScR (Tricco et al., 2020) e no capítulo do Manual do JBI para revisões de escopo (Peters et al., 2020), a seguir, foram elencadas, na ótica dos autores, as limitações desta revisão: Não haver a publicação de um protocolo de revisão, a priori; Considerar apenas estudos publicados nos últimos de 20 anos; Considerar apenas três idiomas (inglês, espanhol e português); Não ter realizado buscas nas listas de referências dos estudos selecionados; Não fornecer o registro da data exata da busca em cada banco de dados; Inclusão de apenas um pequeno número de bancos de dados (fontes de informação). Para ampliar a abrangência da busca, outros bancos de dados como Embase, Web of Science, Scopus, CINAHL e ScienceDirect, bem como bancos de teses e dissertações internacionais, devem ser considerados em revisões futuras sobre o tópico; não haver métodos para minimizar erros na extração de dados, como extração de dados em duplicata ou de forma independente.

\section{Conclusão}

A utilização de joelheira apresenta diversos em indivíduos portadores de OA de joelho e a SDPF, promovendo o alívio da dor e melhora da função do joelho, bem como de: melhora da capacidade de realização de atividades de vida diária, da capacidade funcional, da capacidade de contração voluntária máxima, do equilíbrio; de aumento de amplitude de movimento; de diminuição da inibição muscular artrogênica, de rigidez articular, da dor, do risco de queda; ajuda no controle da inflamação; e além disso, ainda reduz o ângulo e picos de adução do joelho, que são um fator de risco para a progressão dessas condições.

\subsection{Implicações dos resultados para a prática clínica}

De acordo com as evidências analisadas, aconselha-se o uso de joelheiras elásticas (e/ou articuladas), ajustáveis, com reforço patelar. É essencial que portadores de OA e SDPF procurem assistência profissional para saber a prescrição adequada do tipo ideal de joelheira, do tempo de uso e do material do equipamento.

\subsection{Implicações dos resultados da pesquisa}

Aconselha-se que em ensaios clínicos futuros sejam adotadas melhores estratégias de cegamento dos participantes e dos avaliadores, visando produzir trabalhos com uma melhor qualidade metodológica. Além disso, tem-se a necessidade de saber o tempo e duração ideal de uso da joelheira (dose) e qual o melhor tipo de joelheira. Estudos de revisão sistemática, com uso do GRADE (Grading of Recommendations Assessment, Development and Evaluation), são necessários para verificar a certeza dos achados.

\section{Agradecimentos}

Os autores deste estudo agradecem a Deus, YHWH, por proporcionar o desenvolvimento desta revisão.

\section{Referências}

Bersotti, F. M. (2018). Alterações na demanda metabólica no fortalecimento muscular em indivíduos com osteoartrite de joelho. RBPFEX-Revista Brasileira De Prescrição E Fisiologia Do Exercício, 12(75), 418-425.

Bryk, F. F., Jesus, J. F. D., Fukuda, T. Y., Moreira, E. G., Marcondes, F. B., \& Santos, M. G. D. (2011). Efeito imediato da utilização da joelheira elástica em indivíduos com osteoartrite. Revista Brasileira de Reumatologia, 51(1), 440-446.

Callaghan, M. J., Parkes, M. J., \& Felson, D. T. (2016). The effect of knee braces on quadriceps strength and inhibition in subjects with patellofemoral osteoarthritis. journal of orthopaedic \& sports physical therapy, 46(1), 19-25. 
Chacur, E. P., Oliveira, L., Luz, G. C. P., da Silva, P. L., Baraúna, M. A., \& Cheik, N. C. (2017). Obesidade e sua correlação com a osteoartrite de joelho em mulheres. Fisioterapia em Movimento, 21(2).

Charlier, E., Relic, B., Deroyer, C., Malaise, O., Neuville, S., Collée, J., ... \& De Seny, D. (2016). Insights on molecular mechanisms of chondrocytes death in osteoarthritis. International journal of molecular sciences, 17(12), 2146.

Chen, D. I., Shen, J., Zhao, W., Wang, T., Han, L., Hamilton, J. L., \& Im, H. J. (2017). Osteoarthritis: toward a comprehensive understanding of pathological mechanism. Bone research, 5(1), 1-13.

Chuang, S. H., Huang, M. H., Chen, T. W., Weng, M. C., Liu, C. W., \& Chen, C. H. (2007). Effect of knee sleeve on static and dynamic balance in patients with knee osteoarthritis. The Kaohsiung journal of medical sciences, 23(8), 405-411.

Ghasemi, M. S., \& Dehghan, N. (2015). The comparison of Neoprene palumbo and Genu direxa stable orthosis effects on pain and activity of daily living in patients with patellofemoral syndrome: a randomized blinded clinical trial. Electronic physician, 7(6), 1325.

Meira, S. S., Vilela, A. B. A., de Souza, I. M., de Matos, S. B., \& Pestana, A. M. S. (2013). Síndrome da dor femoropatelar: uma revisão sistemática. Brazilian Journal of Biomechanics, 13(25), 71-75.

Melo S. M., Gomes, V. M. D. S. A., do Monte, J. A., Lopes, A. C. B., do Monte, E. J. S., Queiroz, H. E. V., ... \& Uchôa, É. P. B. L. (2021). A influência da osteoartrite de joelho no equilíbrio e marcha de indivíduos idosos. Research, Society and Development, 10(10), e347101018735-e347101018735.

Oliveira, A. M. I. D., Peccin, M. S., Silva, K. N. G. D., Teixeira, L. E. P. D. P., \& Trevisani, V. F. M. (2012). Impact of exercise on the functional capacity and pain of patients with knee osteoarthritis: a randomized clinical trial. Revista Brasileira de Reumatologia, 52(1), 876-882.

Oliveira, L. V. D., Saad, M. C., Felício, L. R., \& Grossi, D. B. (2014). Muscle strength analysis of hip and knee stabilizers in individuals with Patellofemoral Pain Syndrome. Fisioterapia e Pesquisa, 21(1), 327-332.

Peters, M., Godfrey, C., McInerney, P., Munn, Z., Trico, A., \& Khalil, H. (2020). Chapter 11: Scoping Reviews. In E. Aromataris \& Z. Munn (Eds.), JBI Manual for Evidence Synthesis (pp. 407-452). JBI. https://doi.org/10.46658/JBIMES-20-12.

Schween, R., Gehring, D., \& Gollhofer, A. (2015). Immediate effects of an elastic knee sleeve on frontal plane gait biomechanics in knee osteoarthritis. PloS one, 10(1), e0115782.

Shiwa, S. R., Costa, L. O. P., Moser, A. D. D. L., Aguiar, I. D. C., \& Oliveira, L. V. F. D. (2011). PEDro: a base de dados de evidências em fisioterapia. Fisioterapia em Movimento, 24(1), 523-533.

Tavares, G. M. S., de Oliveira Brasil, A. C., Nunes, P. M., Costa, N. L., De Gasperi, G., Piazza, L., \& Santos, G. M. (2011). Condromalácia patelar: análise de quatro testes clínicos. ConScientiae Saúde, 10(1), 77-82.

Tricco, A. C., Lillie, E., Zarin, W., O'Brien, K. K., Colquhoun, H., Levac, D., ... \& Straus, S. E. (2018). PRISMA extension for scoping reviews (PRISMA$\mathrm{ScR})$ : checklist and explanation. Annals of internal medicine, 169(7), 467-473.

Vargas e Silva, N. C. D. O., Cardoso, T. D. S. G., Andrade, E. A. D., Battistella, L. R., \& Alfieri, F. M. (2020). Dor, incapacidade e catastrofização em indivíduos com osteoartrite do joelho. BrJP, 3(1), 322-327.

Yamada, E. F., Müller, F. A., Teixeira, L. P., \& Silva, M. D. D. (2018). Efeito dos exercícios de fortalecimento, de marcha e de equilíbrio no tratamento de osteoartrite de joelho. Revista Brasileira de Ciência e Movimento, 26(3), 5-13.

Yamamoto, G. J., Ocampos, G. P., Luzo, M. C. M., Silva, C. A. C. D., Farias, F. E. S. D., \& Rezende, M. U. D. (2019). Randomized prospective study on the treatment of femoro-patellar osteoarthritis using bracing. Acta ortopedica brasileira, 27(1), 85-91. 